Applied Mathematical Sciences, Vol. 8, 2014, no. 103, 5105 - 5114

HIKARI Ltd, www.m-hikari.com

http://dx.doi.org/10.12988/ams.2014.46428

\title{
Fuzzy Linear Programming \\ for Supply Chain Management in Steel Industry
}

\author{
Mahmoud Alrefaei, Ameen Alawneh ${ }^{1}$ and Mansur Hassan \\ Jordan University of Science and Technology \\ Department of Mathematics and Statistics \\ P.O Box 3030, Irbid 22110, Jordan
}

Copyright (c) 2014 M. Alrefaei, A. Alawneh and M. Hassan. This is an open access article distributed under the Creative Commons Attribution License, which permits unrestricted use, distribution, and reproduction in any medium, provided the original work is properly cited.

\begin{abstract}
Linear programming is one of the frequently applied tools in supply chain management. However, managers and decision makers may lack information about exact values of most of the parameters used in the optimization models. Fortunately, fuzzy linear programming comes up with a powerful tool to deal with this kind of incomplete data. In this paper, the flexible approach of fuzzy linear programming is proposed and used to solve supply chain management of steel manufacturing company. This approach reformulated some constraints from conventional linear programming to fuzzy linear programming and provides alternative solutions to decision makers. The results obtained indicate that the fuzzy linear programming gives more flexibility to the decision maker to achieve some aspiration level in order to choose what he considers as the best optimal solution.
\end{abstract}

Subject Classification: 65K05, 90Cxx

Keywords: Fuzzy Linear Programming; Supply Chain Management

\footnotetext{
${ }^{1}$ Qatar University, Department of Math, Stat and Physics on leave from Jordan University of Science and Technology
} 


\section{Introduction}

Many problems arose concerning the supply chain management with imprecise data. Usually supply chain management problems are formulated as conventional linear programming problems with the objective of optimizing supply, process and/or demand. However, most of the parameters used in the real world are not precise. Even if the results obtained using linear programming is accepted, neither the expected revenues nor the constraints can be characterized by uncertainty. The source of uncertainty are classified into three parameters; demand, process/manufacturing and supply. The most important aspects in dealing with imprecise information is the use of membership functions, the value of the membership function represents the degree of satisfaction (preference) on the constraints. The feasible alternative with the maximum degree of satisfaction is chosen as the optimal solution of the production scheduling problem.

In many cases the available materials to be supplied may not be known precisely or a change may occur in some range because he can get additional materials from suppliers, or the available time may also change. IN these cases Fuzzy Linear Programming (FLP) is the best approach to use instead of the conventional LP. In this paper, we will consider the flexible approach to formulate the FLP. In this approach, the decision variables are crisp numbers while the right hand side coefficient are characterized by uncertainties. The proposed FLP will be implemented on a real supply chain management problem in steel industry.

The theory of fuzzy mathematical programming was first proposed by Tanaka et al. [6] based on the fuzzy decision to address the uncertainty of the parameters in the problems with fuzzy objective functions and constraints. The formulation of FLP was first introduced by Zimmermann [8]. He built a crisp model of the problem and obtained its results by an existing algorithm. He then used the results and fuzzified the problem by evaluating admissible deviations for the goal and constraints of the subjective constants, then he represented the maximization of the minimization of the deviations on the constraints by defining an equivalent crisp problem using auxiliary variable. He used Bellman and Zadeh's [7] interpretation that a fuzzy decision is a union of goals and constraints.

\section{Supply Chain of Steel Industry}

Over the last two decades supply chain management (SCM) has attracted ever increasing attention as a consequence of the pressure on organizations in competitive market place to create and deliver value to customers. According to Min and Zhou [4], a supply chain is an integrated system which synchronizes 
a series of inter-related business processes. Petrovic et al. [5] was the earlier contributors to describe the fuzzy modeling and simulation of a supply chain in an uncertain environment. Carlsson and fuller [2] proposed a fuzzy logic approach to reduce bullwhip effect.

The motivation of this research is to provide an alternative way for optimizing supply chain management in an uncertain environment, which at the end helps the decision makers to take their decision and implement the results obtained. The main aims of this research is to implement FLP on solving supply chain in steel industry, more precisely we are interested in developing a fuzzy linear programming formulation to describe the supply chain, from suppliers to consumers and implement it for solving a supply chain management problems. We then incorporate existing data in the proposed model in order to enhance its validity and ensure practical and optimal solutions.

The overall components of the supply chain problem can be summarized as follows Diabat et al. [3]:

- The number of suppliers that are responsible for supplying the raw materials.

- The cost of raw materials to be supplied.

- The inventory level of any item from suppliers that enters in to inventory. The lower and upper inventory level, minimum and maximum purchased unit, holding cost and duration in inventory are to be considered.

- The products sold; its minimum sold, maximum sold, lower and upper inventory level are to be considered.

- Production plant. Its receiving capacity, production capacity and time before shipping out.

- Customers, which includes distributors, products sold, products lead time/materials and selling price.

Figure 2.1 shows the main elements/stages in a supply chain network from raw materials to customers.

\subsection{Formulating the Problem as Conventional LP}

The supply chain problem has been formulated as a conventional LP problem model Alawneh et. al [1]. However, the purchase of raw materials is not always specific, therefore any raw material constraints can be considered as fuzzy with uncertain at the right hand side. In this case, we can use flexible approach to reformulate the aforementioned constraints to meet the target of the decision 


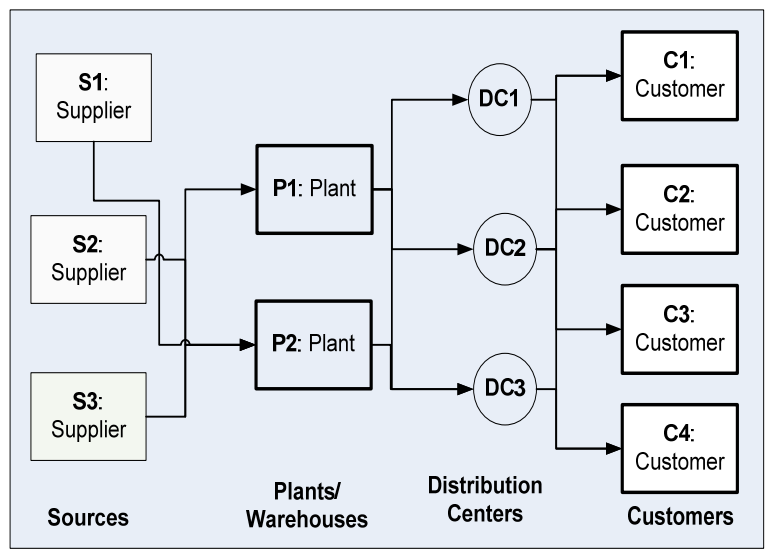

Figure 2.1: Supply chain network.

makers. We reformulate the first two constraints in to Fuzzy constraints and study the effect of the new solution to maximize the company's profit.

consider 4 types of products; namely product1, product2, product3, and product4, 6 customer sites; customer1, customer $2, \ldots$, customer6 and 5 suppliers, namely supplier1, supplier2,..., supplier5. Furthermore, there are 4 processing units, namely Direct Reduction [DR], the Electric Furnace [EF], the Continues Casting [CC] and the Rolling Mill [RM]. In the model we consider the time to be in hour or days, in order to account for duration in the inventory. The flow chart below is provided to illustrate the proposed model.

The decision variables of the proposed model are expressed in tons as follows:

ordo $_{i}$ the order of oxide pellets from supplier $i$

ord $s_{1}$ the order of scrap from supplier 1

ord $s_{2}$ the order of scrap from supplier 2

$o_{i}$ the amount of oxide pellets from supplier $i$ that enters DR

$s_{1}$ the amount of scrap from supplier 1

$s_{2}$ the amount of scrap from supplier 2

$i o_{i}$ the level of oxide pellets from supplier $i$ that enters inventory

$i s_{1}$ the level of scrap from supplier 1 that enters inventory

$i s_{2}$ the level of scrap from supplier 2 that enters inventory

$a_{1}$ the amount of high pure iron that exits DR and enters EF

$a_{2}$ the amount of scrap that enters EF

$b$ the amount of molten steel that exits EF and enters CC

$d$ the amount of product3 that exit $\mathrm{CC}$ and enter RM 
$f_{j}$ the amount of finished product that will be sent to costumer $j$.

Other parameters involved in the model are as follows:

$d r$ capacity of direct reduction.

ef capacity of electric furnace.

$c c$ capacity of continuous casting.

rm capacity of rolling mill.

$p_{j}$ price per unit.

$c_{i}$ unit cost

$c s_{1}$ cost of scrap from supplier 1 .

$c s_{2}$ cost of scrap from supplier 2.

$i c_{i}$ holding cost from supplier i.

The objective function is expressed as follows:

$\max Z=\sum_{j=1}^{n} p_{j} . f_{j}-\sum_{i=1}^{m} c_{i} . o r d o_{i}-\left[s_{1} . c s_{1}+s_{2} . c s_{2}\right]-\sum_{i=1}^{m} i c_{i} . i o_{i}-\left[i c_{3} . i s_{1}+i c_{4} . i s_{2}\right]$

subject to the following constraints:

$$
\begin{gathered}
\text { minord } \leq \sum_{t} \text { ordo } o_{i} \leq \text { maxord } \\
\text { minscrap } \leq \sum_{t} \text { ord } s_{i} \leq \text { maxscrap } \\
a_{1}=\sum_{i=1}^{m} d r_{1 i} \cdot o_{i}+d r_{13} \cdot s_{1}+d r_{14} \cdot s_{2} \\
a_{2}=\sum_{i=1}^{m} d r_{2 i} \cdot o_{i}+d r_{23} \cdot s_{1}+d r_{24} \cdot s_{2} \\
b=e f_{1} \cdot a_{1}+e f_{2} \cdot a_{2} \\
d=c c . b \\
f p=r m . d \\
f_{1}+f_{2} \leq f p \\
i o_{i}=o_{i}-o r d o_{i} \\
i s_{i}=s_{i}-o r d s_{i} \\
\sum_{t} o_{1}+o_{2} \leq d r \\
\sum_{t} a_{1}+a_{2} \leq e f
\end{gathered}
$$




$$
\begin{gathered}
\sum_{t} b \leq c c \\
\sum_{t} d \leq r m
\end{gathered}
$$

The objective of the model aims at maximizing the profit over one year, which consist of the following terms: the income from selling the finished products to consumers, minus the cost of purchasing the raw materials, minus the cost of holding raw materials in inventory.

The first two constraints guarantee that the orders placed for raw materials from all suppliers will be within the given range of minimum and maximum quantities. From these information the first two constraints can be considered as fuzzy constraints with uncertain at the right hand side.

\subsection{Reformulation of the First Two Constraint as Fuzzy}

Let $B_{1}$ be the minimum purchased oxide pellets then the maximum purchased oxide pellets is $B_{1}+\theta_{1}$, again let $B_{2}$ be the minimum purchased scrap then the maximum purchased scrap is $B_{2}+\theta_{2} \theta_{1}$ and $\theta_{2}$ can be obtained by subtracting minimum from the maximum. the constraints (1) and (2) can be reformulated as follows:

$$
\begin{aligned}
\sum_{t} \operatorname{ord}_{i} & =\tilde{B}_{1}, \tilde{B}_{1} \in\left[B_{1}, B_{1}+\theta_{1}\right] \\
\sum_{t} \operatorname{ords}_{i} & =\tilde{B}_{2}, \tilde{B}_{2} \in\left[B_{2}, B_{2}+\theta_{2}\right]
\end{aligned}
$$

$\theta_{1}$ and $\theta_{2}$ are the maximum purchased oxide pellets and maximum purchased scrap tolerance respectively

\subsection{Estimation of Membership Function}

The estimation of the membership functions for the constraints for the first constraints, linear type is assumed.

$$
\mu_{1}\left(\sum_{t} \operatorname{ordo}_{i}\right)= \begin{cases}0 & \text { if } \sum_{t} \text { ordo } o_{i}<B_{1} \text { or } \sum_{t} \text { ord } o_{i}>B_{1}+\theta_{1} \\ 1 & \text { if } B_{1}<\sum_{t} \text { ordo } o_{i}<B_{1}+\theta_{1} \\ \left.1-\frac{\left(\sum_{t}\right. \text { ordoi }}{\theta_{1}}-B_{1}\right) & \text { if } B_{1} \leq \sum_{t} \text { ord } d_{i} \leq B_{1}+\theta_{1}\end{cases}
$$

for the second linear type is also assumed. The membership function for the first constraint is give depicted in Figure 2.2.

$$
\mu_{2}\left(\sum_{t} \text { ords } s_{i}\right)= \begin{cases}0 & \text { if } \sum_{t} \text { ord } s_{i}<B_{2}, \sum_{t} \text { ord } s_{i}>B_{2}+\theta_{2} \\ 1 & \text { if } B_{2}<\sum_{t} \text { ord } s_{i}<B_{2}+\theta_{2} \\ 1-\frac{\left(\sum_{t} \text { ords } s_{i}-B_{2}\right)}{\theta_{2}} & \text { if } B_{2} \leq \sum_{t} \text { ords } s_{i} \leq B_{2}+\theta_{2}\end{cases}
$$

where $\theta_{1}, \theta_{2} \geq 0$. Likewise the membership function for the second constraint is given by Figure 2.3. 


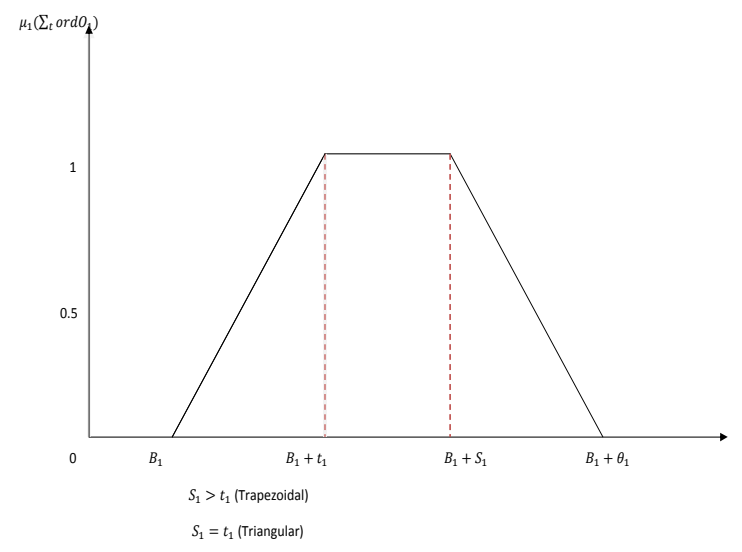

Figure 2.2: Membership function for the first constraint

\subsection{Converting the Problem in to Parametric Linear Programming}

the fuzzy linear programming can be converted to convectional linear programming as follows:

$$
\max Z=\sum_{j=1}^{n} p_{j} . f_{j}-\sum_{i=1}^{m} c_{i} . o r d o_{i}-\left[s_{1} . c s_{1}+s_{2} . c s_{2}\right]-\sum_{i=1}^{m} i c_{i} . i o_{i}-\left[i c_{3} . i s_{1}+i c_{4} . i s_{2}\right]
$$

subject to ;

$$
\begin{aligned}
& \sum_{t} \operatorname{ordo}_{i}=B_{1}+\theta_{1}(1-\alpha) \\
& \sum_{t} \operatorname{ords}_{i}=B_{2}+\theta_{2}(1-\alpha)
\end{aligned}
$$

where $\alpha \in[0,1]$

the other crisp constraints remain the same (i.e. 3 to 14 ), setting $\gamma=1-\alpha$, the constraints can be transformed to the following form

$$
\begin{aligned}
\sum_{t} \text { ordo }_{i} & =B_{1}+\theta_{1} \gamma \\
\sum_{t} \text { ords }_{i} & =B_{2}+\theta_{2} \gamma
\end{aligned}
$$

All the decision variables satisfy the non-negativity and $\gamma \in[0,1]$ is a parameter.

The actual data are from the case company and they are summarized in Tables 2.1, 2.2 and 2.3. 


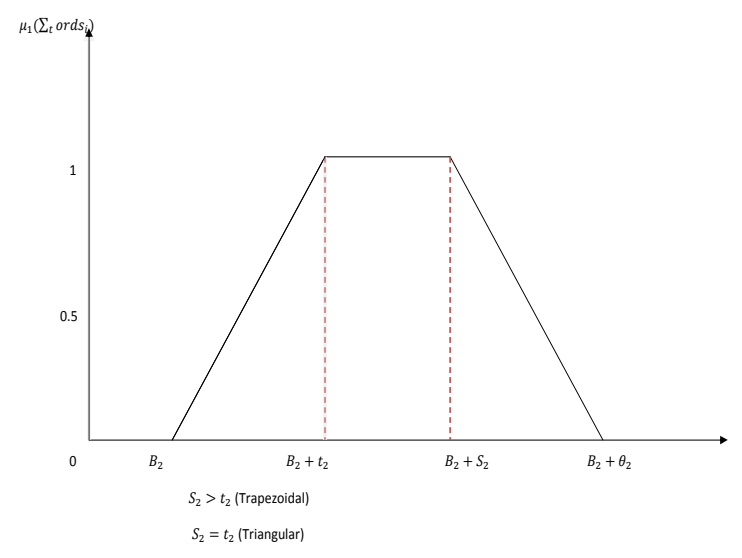

Figure 2.3: Membership function for the second constraint

Table 2.1: Products

\begin{tabular}{|c|c|c|c|c|c|c|}
\hline $\begin{array}{c}\text { Product } \\
\text { Type }\end{array}$ & $\begin{array}{c}\text { Min } \\
\text { Sold (Ton) }\end{array}$ & $\begin{array}{c}\text { Unit Price } \\
\text { (USD/Ton) }\end{array}$ & $\begin{array}{c}\text { Max } \\
\text { Sold (Ton) }\end{array}$ & $\begin{array}{c}\text { Low Inv. } \\
\text { Level (Ton) }\end{array}$ & $\begin{array}{c}\text { Raw } \\
\text { Lever Inv. } \\
\text { Level (Ton) }\end{array}$ & $\begin{array}{c}\text { Material } \\
\text { Per Unit }\end{array}$ \\
\hline Product1 & 120,000 & 420 & 230,000 & 70,000 & 150,000 & 1.45 \\
Product2 & 160,000 & 420 & 350,000 & 30,000 & 60,000 & 1.45 \\
Product3 & 350,000 & 620 & 450,000 & 45,000 & 80,000 & 1.02 \\
Product4 & $1,550,000$ & 725 & $1,637,000$ & 25,000 & 50,000 & 1.01 \\
\hline
\end{tabular}

\section{Results and Analysis}

After reformulation of Steel Company from conventional LP to FLP by converting supply constraints in to fuzzy constraints, we implemented the model in the commercial software GAMS, using available data as parameters and introducing additional parameter that belong to the interval $[0,1]$ called maxtol in the model, it is possible to conduct an analysis regarding the optimal levels of raw materials to be purchased and finished products to be supplied to customers. Assuming that the demand is fixed for each customer and based on this demand the amount of raw materials to be supplied will be decided on, taking in to consideration the processes and the capacity of the units. According to the designed model in the software, there are five suppliers considered and six customers, minimum and maximum inventory levels, as well as unit holding price are drawn from the data sheet and included in the model. The additional two constraints are included to ensure that maxtol is between $[0,1]$. 
Table 2.2: Demand table in Tons

\begin{tabular}{|c|c|c|c|c|c|c|}
\hline Customer/Product & Cust. 1 & Cust. 2 & Cust. 3 & Cust. 4 & Cust. 5 & Cust. 6 \\
\hline product1 & 994,342 & 148,389 & 74,881 & 55,491 & 353,573 & 81,250 \\
product2 & 76,981 & 11,488 & 5,797 & 4,297 & 27,400 & 6,290 \\
product3 & 102,642 & 15,318 & 7,730 & 5,728 & 36,498 & 8,387 \\
product4 & 224,529 & 33,507 & 16,909 & 12,530 & 79,839 & 18,347 \\
\hline
\end{tabular}

Table 2.3: Price table (USD/Tons)

\begin{tabular}{|c|c|c|c|c|c|c|}
\hline Customer/Product & Cust. 1 & Cust. 2 & Cust. 3 & Cust. 4 & Cust. 5 & Cust. 6 \\
\hline product1 & 725 & 533 & 805 & 805 & 730 & 690 \\
product2 & 420 & 320 & 460 & 470 & 430 & 410 \\
product3 & 420 & 308 & 450 & 460 & 420 & 400 \\
product4 & 620 & 455 & 687 & 687 & 640 & 624 \\
\hline
\end{tabular}

Table 3.1 summarizes the amount of oxide pellets to be purchased from supplier $i$ in tons.

Table 3.1: Oxide pellets to be supplied

\begin{tabular}{|c|c|}
\hline suppliers & amount of oxide pellets (Tons) \\
\hline supplier 1 & 500,000 \\
\hline supplier 2 & 486,120 \\
\hline supplier 3 & 500,000 \\
\hline supplier 4 & 500,000 \\
\hline supplier 5 & 500,000 \\
\hline
\end{tabular}

Table 3.2 summarizes the level of scrap to be purchased in tons, inventory of oxide pellets, inventory of scrap and the total time required for given demand to be met.

Finally, the objective value (profit) is $1,567,858,997.00$ USD for a fixed demand.

\section{Conclusion}

In this paper, we have discussed formulating a supply chain management of Steel manufacturing company as a fuzzy linear programming by reformulating some of the constraints. It pays more emphasis on the supply of raw materials, it has been considered as an uncertain. This model has been tested by using data from actual 
Table 3.2: Required inventory level

\begin{tabular}{|c|c|}
\hline inventory & level \\
\hline Scrap to be purchased in tons & 227,350 Tons \\
\hline inventory of oxide pellets & 918,433 tons \\
\hline Inventory of scrap & 50,000 Tons \\
\hline Total time required for given demand to be met. & 31,670 Tons \\
\hline
\end{tabular}

steel Company. The flexible approach of fuzzy formulation is more effective than the conventional LP formulation model. Furthermore, the model provides an alternative to managers and decision makers. The General Algebraic Modeling System (GAMS software) has been used to solve the supply chain problem in this paper.

\section{References}

[1] A. Alawneh, M. Alrefaei, A. Diabat, R. Al-Aomar, M. N. Faisal, An LP Model for Optimizing a Supply Chain Management System for Steel Company. Proceedings of the International MultiConference of Engineers and Computer Scientists, IMECS 2014, March 12 - 14, Hong Kong; 2014. 1162 - 1164.

[2] C. Carlison, and R. Fuller, A fuzzy approach to taming the bullwhip effect. Advance in Computational Inteligence and Learning:Method and Applications, international Series in Inteligent Technologies 2002; 18: 247-262.

[3] A. Diabat, R. Al-Aomar, M. Alrefaei, A. Alawneh, M. Faisal, A Framework for Optimizing the Supply Chain Performance of a Steel Producer. In Proceedings of the 15th International Conference on Enterprise Information Systems 2013; pp:554-562.

[4] H. Min, G. Zhou, Supply chain modelling: Past, present and future, Computers and Industrial Engineering 2002; 43: 231-249.

[5] D. Petrovic, R. Roy, R. Petrovic, Modelling and Simulation of a Supply Chain in an Uncertain Environment. European Journal of Operational Research 1998; 109(2): 299-309.

[6] Tanaka, H., Okuda, T. and Asai, K. On Fuzzy mathematical programming. The journal of cybernetics 1993; 3: 37-46.

[7] Zadeh, L.A. Fuzzy sets. Information and control, 1965; 8(3): 338-353.

[8] Zimmermann H.J. Fuzzy programming and Linear programming with several objective functions. Fuzzy Sets and system 1978; 1: 45-55.

Received: June 7, 2014 